\title{
Indicadores de salud mental en mujeres de zonas migratorias (Zacatecas) con o sin pareja migrante
}

\section{Mental health indicators in women of migratory zones (Zacatecas) with or with- out an immigrant husband}

Carlos Ernesto Acevedo Sánchez

María Dolores García Sánchez Docente

Oliva E. Luis Delgado

Óscar Pérez Veyna

Universidad Autónoma de Zacatecas (UAZ)

\section{Resumen}

La Organización Mundial de la Salud (OMS) reconoce que la migración se ha convertido en un problema de salud pública; al mismo tiempo, se ha identificado el grado de vulnerabilidad de las mujeres que radican en los puntos de origen. Por tal motivo, el objetivo de esta investigación fue conocer la prevalencia de los indicadores de salud mental en mujeres de zonas migratorias (Zacatecas), con pareja migrante o no, y comprobar el efecto protector de tener una pareja no inmigrante. Para ello, se aplicó el cuestionario "Perfil epidemiológico de la mujer con pareja migrante en zona tradicional y emergente de alta incidencia migratoria en el estado de Zacatecas". Se utilizó la categoría de indicadores de salud mental, es decir, una escala tipo Likert, con un alpha de Cronbach de 0.897, una validez de $98.3 \%$, una varianza de 118.636 y una desviación estándar de 10.89201 a una muestra de 279 mujeres de los municipios de Jerez y Juan Aldama; de la población mencionada 144 fueron mujeres con pareja no migrante y 135 mujeres con pareja migrante. A través de los análisis estadísticos de prueba de hipótesis, de independencia, intervalos de confianza, estadísticas de contraste, valor de significancia y $r$ para ambos grupos de mujeres, se encontró que las mujeres con pareja migrante presentan mayor prevalencia en los indicadores de hostilidad y ansiedad, además, el efecto protector de tener una pareja no inmigrante influye en los indicadores de depresión, sensibilidad interpersonal y somatización.

Palabras clave: migración, mujer, pareja íntima, salud mental.

Nota del autor

Carlos Ernesto Acevedo Sánchez, Facultad de Psicología Social. Universidad Autónoma de Zacatecas (UAZ).

La correspondencia en relación con este artículo debe dirigirse a Carlos Ernesto Acevedo Sánchez, Facultad de Psicología Social, UAZ. Jardín Juárez 147, Centro Histórico, Zacatecas Centro, C.P. 98000, Zacatecas, Zac. México.

Dirección electrónica: tana_k8outlook.com 


\begin{abstract}
The World Health Organization (WHO) recognizes that migration has become a public health problem; at the same time has degree the vulnerability of the women in the points of origin. For this reason the objective of this research was to know the prevalence of mental health indicators in women from migratory zones (Zacatecas) with migrant partner or not and to verify the protective effect of having a nonimmigrant partner. For this purpose the questionnaire: epidemiological profile of women with migrant partners in traditional and emergent areas with high migratory incidence in the state of Zacatecas, using the category of mental health indicators which is a Likert scale with an alpha of Cronbach of 0.897 , a validity of $98.3 \%$, a variance of 118,636 and a standard deviation of 10.89201 for a sample of 279 women from the municipalities of Jerez and Juan Aldama, of which 144 were women with nonimmigrant partners and 135 women with migrant couple. Trough statistical analyzes of hypothesis testing, independence, confidence intervals, contrast, statistics significance, and the value of $r$ for both groups of women; finding that women with a migrant partner have a higher prevalence in the indicators of hostility and anxiety and the protective effect of having a nonimmigrant partner influences the indicators of depression, interpersonal sensitivity and somatization.
\end{abstract}

Keywords: immigration, intimate partner, mental health, women.

La migración internacional es un fenómeno bastante complejo y uno de los fenómenos globales más importantes de nuestros días, en especial si se tiene en cuenta que todos los países son puntos de origen y de destino de migrantes. Visto así, son muchos los factores que determinan la migración de mexicanos como la oferta y demanda de fuerza laboral, la tradición migratoria, el programa bracero que estimuló de manera formal la migración laboral hacia los Estados Unidos, etc., lo cual generó reunificación familiar en los lugares de origen (Corona, 2014). En tal contexto, la migración para el Instituto Nacional de Estadística y Geografía (INEGI, 2010) significa el cambio de residencia de una o varias personas de manera temporal o definitiva, con una motivación económica cuyo fin es alcanzar una mejor calidad de vida.

Zacatecas es uno de los estados que ha contribuido de manera significativa al flujo migratorio hacia los Estados Unidos, por lo que los migrantes son una población importante, económicamente activa, y el principal motivo de su emigración es el mejoramiento en la calidad de vida de sus familias. La migración internacional en Zacatecas ha sido un fenómeno tan arraigado que no se puede comprender la economía y la dinámica demográfica del estado sin descartar el fenómeno migratorio. La mayoría de sus municipios y hogares depende, en gran medida, de las remesas que los zacatecanos mandan a sus familiares. La tradición migratoria es tan antigua que ha generado una cultura de 
movilidad profundamente arraigada en los zacatecanos (Corona, 2014).

En el 2009 había cerca de 800 mil zacatecanos radicados de manera permanente en Estados Unidos. Según el INEGI (2010), para el 2010 había 1 millón 112 mil 273 mexicanos en Estados Unidos, de los cuales 832 mil 441 eran hombres; asimismo, se encontraban $31 \mathrm{mil}$ 205 zacatecanos migrantes en Estados Unidos, de ellos 24 mil 615 eran hombres, con 3.8\% de entre 0 y 14 años de edad, $45.7 \%$ de entre 15 a 24 años, $23.8 \%$ de 25 a 34 años, 19\% de 35 a 49 años y $4 \%$ de 50 a 59 años de edad. Alrededor de la mitad de migrantes zacatecanos en Estados Unidos se encontraba en edad reproductiva y laboral.

Es difícil saber la cifra exacta de migrantes de origen mexicano que residen en el vecino país del norte; por ejemplo, el gobierno norteamericano a través de la Oficina del Censo reconoció, en abril del año 2013, a 11.6 millones de personas nacidas en México que vivían en territorio estadounidense; sin embargo, las Naciones Unidas (ONU) indican, según su informe de octubre del 2013, que la cifra de mexicanos residentes en Estados Unidos era de alrededor de 13 millones (Corona, 2014).

La migración hacia los Estados Unidos es, tal vez, uno de los factores más significativos e influyentes en el desequilibrio del bienestar psicológico y el desgaste en la salud mental en miles de familias en México; las mujeres y los niños son los más afectados (López, 2007). El cambio en las necesidades de los miembros de la familia y la tensión en el núcleo familiar son tan grandes que rompen el equilibrio y conllevan a problemas que afectan el funcionamiento individual, familiar y social (Lozano et al., 2011).

La literatura permite inferir que los integrantes de familias de migrantes presentan una mayor probabilidad de manifestar un bienestar emocional bajo, lo que a su vez genera estresores individuales, familiares, sociales y comunitarios, relacionados con respuestas orgánicas asociadas con altos niveles de estrés. Éstos varían desde ansiedad, trastornos afectivos, úlceras, colitis, gastritis o los comúnmente denominados nervios, entre otros (Cabassa et al., 2008 como se citó en González, Méndez, Salvador, \& Moreno, 2015).

Las mujeres con pareja migrante que se quedan en los lugares de origen presentan lumbalgias, cefaleas, hipertensión, nerviosismo, cambios de carácter, cansancio crónico y migrañas; dichos padecimientos con frecuencia se han encontrado en pueblos donde se ha hecho trabajo de campo y, a pesar de tener una causa física, durante varios años se ha sospechado que también pueden estar relacionados con el estrés, la ansiedad y el miedo que a diario viven estas mujeres (López, 2007b).

Existen algunos estudios publicados sobre salud mental en familiares de migrantes, en el caso particular de las mujeres con pareja migrante y su relación con la migración y la salud psicoemocional. Por ejemplo, López (2007b) realizó un estudio con una muestra aleatoria de 235 
mujeres, habitantes de diez comunidades rurales de alta migración del Noreste de Michoacán, donde se aplicaron cuestionarios estandarizados para encontrar casos de ansiedad y depresión; los resultados encontraron tasas al doble del promedio nacional. El $41.72 \%$ de mujeres presenta ansiedad y $54.31 \%$ presenta depresión.

Los problemas emocionales detectados fueron malestar general, ansiedad, tristeza, desánimo, falta de motivación, sentimientos de culpa, depresión y nervios.

En estudios realizados por Garduño (2008 como se citó en Rivera, Obregón, \& Cervantes, 2009) en zonas rurales de Michoacán, señalan que con frecuencia las mujeres de migrantes desarrollan trastornos somáticos y psíquicos a partir del abandono y separación de sus maridos, tales como tensión muscular y lumbalgias, gastritis, dolor de cabeza, insomnio y colitis, con un consiguiente aumento del consumo de sustancias psicoactivas (ansiolíticos, alcohol y drogas ilícitas).

Meza y Cuéllar (como se citó en GonzálezFogoaga, 2010) realizaron en el estado de Michoacán entrevistas a profundidad con 55 mujeres, esposas de migrantes, y encontraron que si bien estas mujeres describen a sus esposos como trabajadores, responsables, buena gente, alegres, hombres valientes que sufren y dan su vida por enviarles dinero a sus hijos y a ellas, también expresan emociones negativas de dolor, tristeza, soledad y falta de apoyo cuando los maridos no están en México.
Por su parte, Castro (2008) entrevistó a quince madres de familia, esposas o compañeras de migrantes internacionales de la Ordeña, una región de alta incidencia migratoria del estado de Zacatecas, de cuyos testimonios aunque se deduce que existe el convencimiento de que es mejor estar temporalmente separados, la mayoría de las informantes relata eventos de depresión o depresión posparto relacionados estrechamente con la situación migrante: largas ausencias con el consecuente distanciamiento físico y, en muchos casos, con el paso de los años, alejamiento sentimental, partos sin apoyo o presencia del cónyuge, estrechez económica, entre otros.

En Zacatecas, García y Pérez (2008) realizaron un estudio en las localidades de Tetillas (Río Grande), La Victoria (Pinos) y Tacoaleche (Guadalupe), y encontraron que la ausencia del padre impacta de manera negativa en la familia, pues a decir de las mismas esposas, llegan a sufrir enfermedades nerviosas y depresión crónica, que no siempre quieren o pueden reconocer. El $88.14 \%$ de las mujeres entrevistadas dijo haber pasado por tristeza o depresión en más de una ocasión en los últimos seis meses.

En otra investigación realizada en Zacatecas por García, Lozano, Luis, Robledo y Zavala (2009), en los municipios de Juan Aldama, Miguel Auza y Tlaltenango, se entrevistaron a madres de familia con pareja migrante, quienes expresaron preocupación por la integración 
familiar debido a la ausencia del esposo, por la conducta rebelde de los hijos, en especial de los adolescentes, así como preocupación por su salud y bienestar físico y psicológico, al tiempo que manifestaron la necesidad de contar con servicios especializados de atención a la salud mental en su lugar de residencia.

Por su parte, Lozano et al. (2011) trabajaron con una muestra de 146 mujeres, habitantes de las cabeceras municipales de Jerez, Francisco R. Murguía, Monte Escobedo, General Pánfilo Natera, Pinos y Villa Hidalgo en el estado de Zacatecas, todas ellas con pareja migrante en EE.UU. En dicho estudio se compararon indicadores de salud mental (depresión, hostilidad, sensibilidad interpersonal, somatización y ansiedad), y se registraron diferencias estadísticamente significativas en la subescala de ansiedad, donde las mujeres de la cabecera municipal General Pánfilo Natera fueron quienes presentaron un rango promedio más alto $(84.89) \mathrm{y}$, por lo tanto, mayores niveles de ansiedad.

Una investigación de Arciniega (2012), la cual mide la prevalencia de los indicadores de depresión en 296 mujeres con pareja migrante de Jerez, Francisco R. Murguía, Monte Escobedo, Gral. Pánfilo Natera, Pinos y Villa Hidalgo en el estado de Zacatecas, en ella se encontró una alta prevalencia en tres de los cinco indicadores de depresión; factores como la ausencia del cónyuge, escolaridad, jefatura familiar $\mathrm{y}$ problemas para dormir son factores que pueden incidir en dicha prevalencia.
Por otra parte, Escareño (2012) identifico la prevalencia en los indicadores psicosomáticos en 296 mujeres con pareja migrante de Jerez, Francisco R. Murguía, Monte Escobedo, Gral. Pánfilo Natera, Pinos y Villa Hidalgo en el estado de Zacatecas y encontró una alta prevalencia en dos de los cinco indicadores, en consecuencia, las zonas de alta incidencia migratoria, la edad y el estado legal del cónyuge son factores que pueden repercutir en dicha prevalencia.

La investigación realizada por Acevedo (2016), con base en una muestra de 279 mujeres de zonas de alta incidencia migratoria del estado deZacatecas (144 mujeres con pareja no migrante y 135 mujeres con pareja migrante), sobre indicadores de conductas hostiles, halló que las mujeres con pareja migrante presentan mayor prevalencia, donde la ausencia del cónyuge es un factor que puede incidir en dicha prevalencia. Por ende, en la presente investigación se plantean las siguientes preguntas: ¿qué grupo de mujeres (pareja no migrante y pareja migrante) de zonas migratorias de Zacatecas presentará mayor prevalencia en los indicadores de salud mental?, y ¿el efecto protector de tener una pareja no inmigrante puede influir en la frecuencia de dicha prevalencia?

\section{Objetivos generales de la investigación}

1. Conocer la prevalencia de los indicadores de salud mental en mujeres de zonas migratorias (Zacatecas) con pareja migrante o no. 
2. Comprobar el efecto protector de tener una pareja no inmigrante sobre los indicadores de salud mental.

\section{Hipótesis del estudio}

1. Las mujeres con pareja migrante de zonas migratorias de Zacatecas presentarán mayor prevalencia en los indicadores de salud mental.

2. El efecto protector de tener una pareja no inmigrante influye en la prevalencia de los indicadores de salud mental.

\section{Método}

\section{Participantes}

Las mujeres que participaron en este estudio tienen una característica fundamental: ser compañeras de migrantes, cuya pareja hubo emigrado de su comunidad (Jerez, Los Haro, Juan Aldama y Gral. Juan José Ríos) a los Estados Unidos, ya sea por primera vez o subsiguientes, mientras que ellas se quedaron en las comunidades de origen. Con un grupo de comparación caracterizado por mujeres que residen en Jerez, Los Haro, Juan Aldama y Gral. Juan José Ríos, cuyos esposos no hubieron emigrado; ambos grupos con edades mayores de 18 años. La muestra quedó conformada por 279 mujeres de las zonas de alta incidencia migratoria del estado de Zacatecas (144 mujeres con pareja no migrante y 135 mujeres con pareja migrante), seleccionadas mediante el procedimiento de muestreo no probabilístico a través de la técnica "bola de nieve".

\section{Instrumentos}

El instrumento que se utilizó para recoger los datos de la investigación fue el cuestionario "Perfil epidemiológico de la mujer con pareja migrante en zona tradicional y emergente de alta incidencia migratoria en el estado de Zacatecas", el cual consta de 115 reactivos organizados en sub-escalas. Para efectos de este estudio, se utilizó la categoría de indicadores de salud mental, es decir, una escala tipo Likert, dividida en las subescalas: depresión $(\alpha=.85)$, hostilidad $(\alpha=.83)$, sensibilidad interpersonal $(\alpha=.84)$, somatización $(\alpha=.80)$ y ansiedad $(\alpha=.84)$. Presenta un alpha de Cronbach de 0.897, con una validez de $98.3 \%$, una varianza de 118.636 y una desviación estándar de 10.89201. El instrumento resulta altamente confiable y viable en su aplicación y corresponde a la adaptación que hicieron Sandín, Valiente, Chorot, Santed y Lostao (2008) del Symptom Assessment-45 Questionnaire (SA-45), que es un instrumento de síntomas psicopatológicos de 45 ítems.

\section{Procedimiento}

1. Se aplicó la versión abreviada del cuestionario Symptom Assessment-45 Questionnaire (SA45), el cual es un autoinforme de síntomas psicopatológicos, que consta de 45 reactivos, divididos en nueve subescalas: depresión, hostilidad, sensibilidad interpersonal, somatización, ansiedad, psicoticismo, obsesión-compulsión, ansiedad fóbica e ideación paranoide. Adaptado por Lozano 
et al. (2011) en la investigación que lleva como nombre: Indicadores de salud mental en mujeres de Zacatecas con pareja migrante; después de pilotear el instrumento quedó conformado por las siguientes escalas: depresión, hostilidad, sensibilidad interpersonal, somatización y ansiedad.

2. La muestra se obtuvo con base en el total de casos para ambos grupos de mujeres que estuvieron dispuestos a participar en la investigación.

3. Se procesó la información de los cuestionarios a través del programa estadístico SPSS Statistics, versión 21 para Windows 10 , con el fin de obtener así la frecuencia de los indicadores de salud mental para ambos grupos de mujeres.

4. Se realizaron los análisis estadísticos a través de las pruebas siguientes: prueba de hipótesis, de independencia, intervalos de confianza, estadística de contraste, valor de significancia y $r$ para ambos grupos de mujeres en los indicadores de salud mental.

\section{Resultados}

Las mujeres con pareja no migrante y pareja migrante de zonas de incidencia migratoria del estado de Zacatecas se caracterizan por tener un rango de edad de los 18 hasta los 60 años y más; las mujeres con pareja no migrante presentan una mayor proporción de los 40-49 años, y las mujeres con pareja migrante presentan un rango de los 30-39 años, ambos grupos de mujeres tienen una escolaridad de secundaria completa y conciben a su pareja como el jefe de familia, habitan en casa propia donde llegan a vivir de una a cuatro personas y tienen de tres a cinco, $y$ de uno a dos hijos, respectivamente; son amas de casa y cuentan con Seguro Popular.

En la tabla 1 se registra la prevalencia de los indicadores de salud mental para mujeres con pareja migrante y no de zonas de incidencia migratoria (Zacatecas).

Tabla 1

Propiedades psicométricas de mujeres con pareja inmigrante y no de zonas de incidencia migratoria (Zacatecas) para los indicadores de salud mental

\begin{tabular}{lcccccccccc}
\hline & \multicolumn{1}{c}{ Depresión } & \multicolumn{2}{c}{ Hostilidad } & \multicolumn{2}{c}{$\begin{array}{c}\text { Sensibilidad } \\
\text { interpersonal }\end{array}$} & Somatización & \multicolumn{2}{c}{ Ansiedad } \\
\hline & pnm & Pm & pnm & pm & pnm & Pm & pnm & pm & pnm & pm \\
\hline Nada & 64 & 55 & 77 & 67 & 67 & 67 & 53 & 50 & 62 & 49 \\
Poco & 29 & 32 & 21 & 24 & 27 & 24 & 36 & 35 & 29 & 32 \\
Mucho & 8 & 13 & 2 & 9 & 6 & 9 & 11 & 15 & 9 & 18 \\
\hline Total & $100 \%$ & $100 \%$ & $100 \%$ & $100 \%$ & $100 \%$ & $100 \%$ & $100 \%$ & $100 \%$ & $100 \%$ & $100 \%$ \\
\hline
\end{tabular}

Fuente: Datos obtenidos de la adaptación del Symptom Assessment-45 Questionnaire (SA-45).

Nota: pareja no migrante (pnm) pareja migrante (pm). 
En la tabla 2 se presentan los resultados para las pruebas estadísticas de distribución normal estándar y chi cuadrada para independencia en el caso de ambos grupos de mujeres.

Las mujeres con pareja migrante de zonas migratorias de Zacatecas registran mayor prevalencia en los indicadores de hostilidad y ansiedad.
En la tabla 3 se presentan los resultados para el cálculo de intervalos de confianza, estadística de contraste, valor de significancia y $r$ para ambos grupos de mujeres en los indicadores de salud mental.

Tabla 2

Pruebas de hipótesis e independencia para los indicadores de salud mental en mujeres de zonas de incidencia migratoria (Zacatecas)

Prueba de hipótesis

Distribución normal estándar

\begin{tabular}{|c|c|c|c|c|c|c|}
\hline Indicador & Estadística & $\begin{array}{l}\text { Tabla } \\
\text { estadística }\end{array}$ & Conclusión & Estadística & $\begin{array}{l}\text { Tabla } \\
\text { estadística }\end{array}$ & Conclusión \\
\hline Depresión & $Z c=1.56$ & 1.64 & No rechazo Ho & $X_{2}=3.395$ & 5.991 & No rechazo Ho \\
\hline Hostilidad & $Z_{c}=2.72$ & 1.64 & Rechazo Ho & $X_{2}=8.249$ & 5.991 & Rechazo Ho \\
\hline $\begin{array}{l}\text { Sensibilidad inter- } \\
\text { personal }\end{array}$ & $Z_{c}=.83$ & 1.64 & No rechazo Ho & $X_{2}=.847$ & 5.991 & No rechazo Ho \\
\hline Somatización & $Z_{c}=.92$ & 1.64 & No rechazo Ho & $X_{2}=.834$ & 5.991 & No rechazo Ho \\
\hline Ansiedad & $\mathrm{Zc}=2.36$ & 1.64 & Rechazo Ho & $X_{2}=7.479$ & 5.991 & Rechazo Ho \\
\hline
\end{tabular}

Tabla 3

Intervalos de confianza, estadística de contraste, valor de significancia y r para ambos grupos de mujeres en los indicadores de salud mental

\begin{tabular}{lccccc}
\hline \multicolumn{1}{c}{ Indicador } & \multicolumn{2}{c}{$\begin{array}{c}\text { Intervalos de confianza } \\
(95 \%)\end{array}$} & $\begin{array}{c}\text { Contraste } \\
\text { Ho: } p_{1}=p_{2} \\
\text { Ha: } p_{1} \neq p_{2}\end{array}$ & Significancia & $r=Z /(N)_{1 / 2}$ \\
\hline Depresión & $\mathrm{Pm}$ & $\mathrm{pnm}$ & & & \\
Hostilidad & {$[.070 ; .18]$} & {$[.04 ; .12]$} & No R Ho Zc= $1.37 \mathrm{~N} . \mathrm{S}$ & $\mathrm{P}>.05$ & 0.08 \\
Sensibilidad Interpersonal & {$[.042 ; .138]$} & {$[0.0 ; .04]$} & $\mathrm{R}$ Ho Zc= $2.61 *$ & $\mathrm{P}<.05$ & 0.16 \\
Somatización & {$[.042 ; .138]$} & {$[.03 ; .09]$} & No R Ho Zc=.95 N.S & $\mathrm{P}>.05$ & 0.05 \\
Ansiedad & {$[.09 ; .210]$} & {$[.06 ; .16]$} & No R Ho Zc=.30 N.S & $\mathrm{P}>.05$ & 0.01 \\
\hline
\end{tabular}

Nota: pareja no migrante (pnm), pareja migrante (pm).

N.S: no significativa *diferencia significativa. 
Tras elaborar las pruebas estadísticas pertinentes para cada uno de los indicadores de salud mental en ambos grupos de mujeres, se encontró significativa la migración con los siguientes indicadores: al elaborar los intervalos de confianza para el indicador de hostilidad con una confianza de 95\%, el grupo de mujeres con pareja migrante oscila entre (.042 y .138\%), mientras que en mujeres con pareja no migrante se ubica entre $(.00 \mathrm{y} .138 \%)$. Al realizar el contraste de significancia entre ambos grupos, se rechazó de la hipótesis de igualdad de proporciones $(\mathrm{P}<.05)$ y un cálculo de $r=.16$, lo cual indica que el efecto de la migración sobre la hostilidad es significativo.

Mientras que para la ansiedad, los intervalos de confianza para el grupo de mujeres con pareja migrante oscilan entre (.177 y .182\%), y en mujeres con pareja no migrante está entre $(.044$ y $.136 \%)$. Al realizar el contraste de significancia entre ambos grupos, se rechazó la hipótesis de igualdad de proporciones $(\mathrm{P}<.05)$ y un cálculo de $r=.19$; lo cual indica que el efecto de la migración sobre la ansiedad es significativo. Por lo tanto, el efecto protector de tener una pareja no inmigrante influyó en la prevalencia de los indicadores de depresión, sensibilidad interpersonal y somatización en mujeres de zonas de incidencia migratoria del estado de Zacatecas.

\section{Discusión}

En la presente investigación se conoció la prevalencia de los indicadores de salud mental en mujeres con pareja migrante y no de zonas de incidencia migratoria de Zacatecas, y se encontró que la mujer con pareja migrante presenta mayor prevalencia en los indicadores de hostilidad y ansiedad, además, el efecto protector de tener una pareja no inmigrante influyó en los indicadores de depresión, sensibilidad interpersonal y somatización.

Lo anterior se puede explicar con base en estudios demográficos, los cuales han demostrado cómo ante la ausencia del cónyuge, las mujeres que se quedan en el lugar de origen asumen una gran cantidad de responsabilidades y las consecuencias de sus decisiones; cambian sus patrones de conducta; administran las remesas, y los recursos familiares; velan por la educación y formación de sus hijos, el cuidado de la parcela; la negociación con la familia política; el constante acoso de los hombres de su comunidad; la participación en asambleas; la incertidumbre ante el comportamiento sexual de su marido en los Estados Unidos, y un largo etcétera como fuentes de estrés, ansiedad y problemas físico-emocionales. Asimismo, facilitan la migración masculina, se preocupan por el desplazamiento de su pareja y la precariedad de su empleo en los Estados Unidos (López, 2007).

Las mujeres en dicha situación desempeñan roles andróginos, al asumir el papel de padre y madre al mismo tiempo. Ante la irregularidad 
o carencia del envío de remesas, se ven obligadas a salir del espacio privado en busca de actividades remuneradas, lo cual les genera dobles y hasta triples jornadas laborales, además de la administración de la parcela, el ganado y el pequeño comercio familiar, por ende, limitan su tiempo libre y postergan sus necesidades para satisfacer las de los demás; aunado a esto, carecen de acceso a los servicios de salud, educación y empleo (Castro, 2008).

Los resultados de esta investigación coinciden con las investigaciones de López (2007b) quien descubrió un alto porcentaje de mujeres michoacanas con pareja migrante con ansiedad (41.72\%) y problemas emocionales como malestar general, ansiedad, tristeza, desánimo, falta de motivación, sentimientos de culpa, depresión y nervios; el estudio de Lozano et al. (2011), encontró, por su parte, altos niveles de ansiedad en mujeres con pareja migrante de la cabecera municipal de General Pánfilo Natera en Zacatecas.

El estudio de Acevedo (2016) demuestra que la ausencia del cónyuge es un factor que puede incidir en la prevalencia de los indicadores de hostilidad en mujeres de zonas de alta incidencia migratoria del estado de Zacatecas. En tanto Castro (2008), indica que estas mujeres presentan conductas hostiles hacia sus hijos como ira y violencia física, debido a la migración de su esposo, la misma crianza de los hijos, la manutención, el aseo de la casa, la administración de los recursos, entre otros aspectos.
Zacatecas, al ser una entidad con una larga tradición migratoria, ha influido para que las mujeres con pareja migrante se hayan hecho a la idea de su situación, por ende, sobrellevan los problemas psicológicos que conllevan el distanciamiento físico y las largas ausencias de sus parejas; estas mujeres han logrado sobrellevar sus malestares y adecuarse a su nuevo estilo de vida, donde al parecer su salud mental se ha ido deteriorando y con los años ha aparecido un mecanismo de adaptación y sublimación ante la migración de sus parejas. Esto ha permitido que los síntomas del desgaste físico y emocional sean perecederos y se desvanezcan a través del tiempo.

En cuanto a las limitaciones del estudio, la técnica de muestreo resultó ser muy tardada y limitante, debido a que cada mujer sólo nos permitía acceder a máximo tres o cuatro mujeres con pareja migrante. Existía poca disposición por parte de las mujeres a causa de falta de tiempo libre. El instrumento resultó ser muy cansado y tardado tanto para el encuestador como para las encuestadas. Además, transcribir la información de los instrumentos a la base de datos resultó ser sumamente tedioso debido a la gran cantidad de ítems por cada cuestionario.

A partir de los resultados hallados en esta investigación se puede concluir que las mujeres con pareja migrante presentan mayor prevalencia en los indicadores de hostilidad $\mathrm{y}$ ansiedad, y el efecto protector de tener una pareja no inmigrante influyó en la prevalencia de los indicadores de depresión, sensibilidad 
interpersonal y somatización en mujeres de zonas de incidencia migratoria (Zacatecas) con pareja migrante o no. Por consiguiente, para futuras investigaciones se sugiere ampliar la cantidad de zonas de alta incidencia migratoria; aplicar entrevistas a profundidad con un estudio longitudinal de la salud mental en mujeres con pareja migrante, tomando como punto inicial la partida del cónyuge; aumentar la cobertura y calidad de los servicios de salud y la cantidad de investigaciones enfocadas a las mujeres con pareja migrante; e implementar modelos más integrales para entender las consecuencias que acarrea el fenómeno migratorio en las familias.

Se sugiere el aumento en la cobertura de los servicios de salud, especialmente de atención psicológica, abrir espacios de participación para la mujer como lo son los grupos de autoayuda, actividades recreativas, talleres, conferencias, terapia psicológica y mejores políticas públicas para este sector. En sí, contar con un lugar donde se les pueda brindar apoyo psicológico tanto a ellas como a sus hijos para enfrentar sus problemas cotidianos.

Las aportaciones que deja este trabajo generan nuevos dividendos y retoman aspectos que han sido descuidados por nuestra propia área, resulta interesante integrar un estudio desde esta perspectiva, ya que no existen datos suficientes que den un panorama general sobre salud mental en mujeres con pareja no migrante y pareja migrante de zonas de alta incidencia migratoria de nuestro estado. Asimismo, se amplió el panorama de la migración, específicamente en familiares que se quedan en su lugar de origen. No obstante, aún falta mucho por conocer y las puertas para seguir buscando, analizando y comprendiendo están abiertas. Proponemos a los psicólogos buscar estrategias y enfoques multidisciplinarios que impliquen parámetros y aportaciones que con el fin de cumplir las demandas y necesidades de la población, por esta razón se exhorta a expandir la línea de investigación en el estado.

\section{Referencias}

Acevedo, C. (2016). Conductas hostiles en mujeres de zonas de alta incidencia migratoria del Estado de Zacatecas. (Tesis de Licenciatura). Zacatecas: Universidad Autónoma de Zacatecas.

Arciniega, E. (2012). Indicadores de depresión en mujeres con pareja migrante de zonas tradicional y emergente de alta incidencia migratoria en el estado de Zacatecas. (Tesis de licenciatura). Zacatecas: Universidad Autónoma de Zacatecas.

Castro, A. (2008). Migración y la salud de las esposas o compañeras de los migrantes de la localidad de la Ordeña, Jerez, Zacatecas. (Tesis de maestría). Zacatecas: Universidad Autónoma de Zacatecas.

Corona, V. (2014). Aproximación al cálculo del saldo neto migratorio en México, 1990-2010. En J. Serrano (coord.). Región y contexto en la encrucijada actual. (pp. 159-177). México: UAZ. 
Escareño, N.(2012). Indicadores psicosomáticos en mujeres con pareja migrante en zona tradicional y emergente de alta incidencia migratoria en el estado de Zacatecas. (Tesis de licenciatura). Zacatecas: Universidad Autónoma de Zacatecas.

García, R., \& Pérez, O. (2008). Un pasivo: Mujeres y niños en comunidades de alta migración internacional en Michoacán, Jalisco y Zacatecas, México. New York: United Nations Children's Fund (UNICEF).

García, S., Lozano, R., Luis, D., Robledo, M., \& Zavala, R., (2009). Indicadores y necesidades psicosociales en zonas migrantes (tradicional y emergente) del estado de Zacatecas. Informe de Investigación. Zacatecas: Universidad Autónoma de Zacatecas.

González-Fagoaga, J. (2010). Reseña de "La vulnerabilidad de los grupos migrantes en México" de Liliana Meza González y Míriam Cuéllar Álvarez. Migraciones Internacionales, 5(3), 233-239. Recuperado dehttp://www.redalyc.org/articulo.oa? $\mathrm{id}=15112895010$.

González, I., Méndez, M., Salvador, C., \& Moreno, M. (2015). El síndrome de Penélope: Reflexiones sobre algunos indicadores del bienestar emocional. Región y Sociedad, 27(63), 249-277. Recuperado de http:// regionysociedad.colson.edu.mx:8085/index. php/rys/article/view/243/229

Instituto Nacional de Estadística y Geografía. (2010). Censo de Población y Vivienda
2010. INEGI. Recuperado de http://www. censo2010.org.mx

López, G. (2007). El síndrome de Penélope. Salud emocional, depresión y ansiedad de mujeres de migrantes. Seminario permanente sobre migración internacional. Tijuana, B.C. 9 marzo de 2007. Recuperado dehttp://www.colef.mx/sepmig/wpcontent/uploads/2013/05/1 era-GustavoL\%C3\%B3pez-El-s\%C3\%ADndrome-dePen\%C3\%A9lope.pdf

López, G. (2007b). Migración, mujeres y salud emocional. Decisio, 18(1), 46-50. Recuperado de http:/www.crefal.edu.mx/ decisio/images/pdf/decisio_18/decisio18_ saber8.pdf

Lozano, G., García, M., Luis, O., Zavala, J., Miramontes, S., \& Sánchez, J. (2011). Indicadores de salud mental en mujeres de Zacatecas con pareja migrante. Revista Mexicana de Investigación en Psicología Social y de la Salud., 3(1), 41-50. Recuperado de http://www.psicumex.uson.mx/revistas/ articulos/1-r1_art4.pdf

Rivera, M., Obregón, N., \& Cervantes, E. (2009). Recursos psicológicos y salud: Consideraciones para la intervención con los migrantes y sus familias. Michoacán: Universidad Michoacana de San Nicolás de Hidalgo.

Recibido el 10 de abril de 2017

Revisado el 28 de abril de 2017

Aceptado el 27 de mayo de 2017 